\title{
Are we being Myopic about Myopia Control?
}

Janis B. Orr PhD, BSc(Hons), MCOptom, DipTp(IP), PGCertHE, FHEA

James S. Wolffsohn BSc(Hons), PgCertHE, PgDipAdvClinOptom, MBA, PhD, PFHEA, FSB, FCOptom, FAAO, Diplomate-CCLRT, FIACLE, FBCLA

Aston University, Aston Triangle, Birmingham B4 7ET, United Kingdom

E-mail address: j.orr@aston.ac.uk

In the August 2014 issue of Contact Lens and Anterior Eye, Kate L. Johnson [1] asked "are we being myopic about myopia control?" In this thought-provoking editorial, Kate Johnson identified the hesitation to fully embrace myopia management amongst her clinical colleagues, and the frustration that this caused her academic colleagues. Is her portrayal of myopia control resistance in clinical practice a fair representation of myopia control worldwide? According to a global survey investigating myopia management attitudes and strategies in clinical practice [2] published in this issue, the answer is 'yes'.

An internet-based questionnaire, containing questions regarding awareness of myopia prevalence, and perceived efficacy and uptake of myopia control strategies, was created by the myopia group at Aston University, and distributed by the BCLA and other professional bodies to eye-care practitioners around the world [2]. Although practitioners are largely aware of the increasing prevalence of myopia and the management strategies available, the majority $(67.5 \pm 37.8 \%)$ prescribe single vision spectacles or contact lenses as their primary mode of myopic correction. This is the first survey of its kind to evaluate worldwide myopia management and, although the results are unsurprising, they confirm our suspicions that the information from scientific literature is not translating to clinical practice. The results of the survey call for clear guidelines for myopia control.

Myopia prevalence has increased throughout the world, particularly in East and South-East Asia, where it has reached epidemic levels. However, it is as prevalent as $20-50 \%$ in US and Europe. Genetics of a child are known to pre-determine their subsequent refractive status, for example, children with two myopic parents are six times more likely to become myopic themselves than children with one or no myopic parents [3]. Despite this, genetics alone cannot account for the rapid increase in myopia prevalence. Several environmental mechanisms have been proposed including: insufficient/inaccurate accommodative response, excessive time spent reading and/or being indoors. Furthermore, animal work has suggested that a relatively hyperopic peripheral refraction drives the excessive eye growth responsible for myopia development/progression.

Despite the large body of research dedicated to understanding the mechanism of excessive eye growth, the exact mechanisms responsible for myopia development and progression are not yet fully understood, presumably due to the multifactorial nature of the disease. In the absence of a single causative factor, several intervention strategies have been investigated, the most promising of which involve contact lens wear.

We know, from the global myopia management survey [2], that single vision spectacles and contact lenses are the most frequently prescribed modality. It has been suggested that this approach increases relative peripheral hyperopic defocus, which, according to animal work, stimulates eye 
growth. Other authors, however, question that peripheral refraction is the primary driver of eye growth, as they reported that some children do not display relative peripheral hyperopia before becoming myopic. Regardless of whether, or not, you buy into the peripheral refraction theory, it is unlikely that single vision spectacles or contact lenses mitigate myopia onset or progression.

Under-correction, the deliberate reduction of the myopic correction, was hypothesised to reduce accommodative demand and induce myopic defocus, which should prevent eye growth. On the contrary, under-correction has been found to accelerate the rate of myopia progression.

Pharmaceutical therapies, such as atropine and pirenzepine, have proven to be highly effective. Atropine in particular has the ability to reduce myopia progression by up to $77 \%$ [4]. Despite this promising figure, a lack of consensus exists for the mechanism of action, the optimum effective dose which minimises unwanted side-effects and the inevitable rebound effect following the cessation of treatment. Another obstacle exists in that neither atropine nor pirenzepine are licensed for myopia control, which is likely to cause further reluctance to prescribe off-label to paediatric patients. Furthermore, low-dose preparations $(<0.5 \%)$ are not commercially available.

It has been established that increased time spent outdoors has a protective effect against myopia development/progression. It is not yet clear why outdoor activity has this effect but it is postulated that bright light triggers the release of dopamine, a retinal transmitter which is believed to prevent eye growth. Other theories suggest that the sunlight itself could play a role, increasing exposure to vitamin D, which has been shown to reduce eye growth. Finally, the increased viewing distances and the high luminance levels afforded by outdoor activities diminish accommodative demand and reduce pupil diameter/increase depth of focus, thereby increasing retinal image quality.

Multifocal spectacles and contact lenses have been shown to reduce myopia progression by reducing hyperopic blur brought about by accommodative lag. Multifocal contact lens designs have been more effective than spectacle lens designs. This is thought to be related to the consistency of near portion alignment and wearing time. In this issue Pauline Kang and colleagues investigate the changes in visual function in these multifocal soft contact lenses when they are worn by young adults [9].

Orthokeratology was introduced to eliminate refractive error or to reduce it to a sufficiently small degree that the patient can function without spectacles or contact lenses for most of the waking day, however in recent years, it has been shown to be effective in the prevention of myopia progression. Although the mechanism by which orthokeratology reduces myopia progression has not been firmly established, it is widely assumed that the mid- peripheral thickening, brought about by the re-distribution of epithelial cells as a result of the central flattening of the lens, increases the power of the mid periphery, effectively reducing peripheral hyperopic defocus.

Orthokeratology studies have yielded consistent levels of myopia retardation of around $50 \%$ [2]. Despite the efficacy of orthokeratology, and the obvious lifestyle benefits it offers, it only constitutes $1 \%$ of new CL fits worldwide. This is presumably due to concerns about contact lens related complications, however the risks of ocular disease in later life due to myopia are greater than the risk of severe contact lens complications. The article by Lee Jong Soo in this issue of Contact Lens and Anterior Eye examines the effect of orthokeratology in patients with myopic regression after refractive surgery, another potential benefit for this lens modality [10]. 
Non-pharmaceutical myopia control has been shown to reduce myopia progression by between 20 and $50 \%$ [2]. Although on the face of it, these figures appear modest, recent papers have put these values into context. Brennan [5] predicted that a $33 \%$ reduction in myopia progression would reduce the frequency of myopia $>-5 \mathrm{D}$ by $73 \%$. If the myopia reduction increased to $50 \%$; the frequency of myopia >-5D was reduced to $90 \%$. These are powerful statistics that strengthen the case for adopting myopia control.

All too often, myopia is regarded as a minor inconvenient correctable refractive problem, which only becomes pathological when it exceeds an arbitrary cut-off of -6D [6]. Myopia <-6D is traditionally considered to be 'physiological', however Flitcroft [6] challenged this view by suggesting that there is no such thing, as all myopia has the potential to become 'pathological'. Myopia within the so-called 'physiological range' represents a risk factor for ocular disease that is comparable with the risks associated with hypertension for cardiovascular disease. The myopia risks for glaucoma and cataract were also comparable with the risks of stroke from smoking $>20$ cigarettes a day. For retinal detachment and myopic maculopathy, myopia carries a risk far in excess of any identified population risk factor for cardiovascular disease. When you look at it from a public health perspective, it seems negligent not to attempt to reduce myopia progression as the odds ratio for developing ocular complications increases sharply with each dioptre of myopia.

According the myopia global myopia management survey [2], the most common reasons practitioners gave for not adopting myopia control strategies related to concerns about efficacy, safety and cost. Those practitioners who only prescribed single vision spectacles or contact lenses reported that there was inadequate information available to them.

The myopia management day at the 2015 BCLA Clinical Conference in Liverpool provided delegates with an excellent opportunity to appreciate the pros and cons of each myopia intervention strategy. The session climaxed with an expert panel debating the future of myopia control. The panel contained leading myopia researchers from around the world, who presented the cases for each intervention strategy. Organisers of other clinical conferences have also acknowledged the importance of myopia control, and placed myopia researchers/experts centre-stage. Although this is a positive step in getting the message across to the pro-active clinicians at the cutting-edge of the profession in attendance, it is not reaching other clinicians who do not attend clinical conferences or engage with the scientific literature. As the majority of clinicians fall into the latter category, is it any wonder that resistance to engage in myopia control is commonplace?

Other papers in this issue of Contact Lens and Anterior Eye could also show potential in the realm of myopia control. Anisa Mahomed et al show how lenses could be manipulated to release pharmaceuticals into the eye, such as low dose atropine [11]. Scleral lenses as reviewed by Walker and colleagues and Sarah La Porta Weber could provide even better stability of lens optics than soft design and so may have a potential role in myopia control $[12,13]$. Minimising dry eye effects such as those affected by hormone replacement investigated by Li Hu and co-workers is essential to compliance with contact lens treatment modalities [14]. Perhaps light transmission and assessed by José $M$ Artigas could contribute to the outdoor environmental myopia progression retardation effects [15]. Who knows, future research may identify Traditional Chinese Medicine herbs such as those examined by Maureen Boost, may prove beneficial [16]. 
As the global myopia management survey [2] suggests, clear clinical management guidelines would be useful so that all myopic patients can be managed consistently and effectively, decreasing their relative risk of progression and/or complications and increasing their quality-of-life. The UK Myopia Consortium, which is led by Dr Nicola Logan, Senior Lecturer at Aston University, and includes myopia researchers from across the UK, is currently working towards this goal. Aston University will also host the 2017

International Myopia Conference (IMC), a biennial research conference attended by world-leading myopia researchers. The IMC will showcase the latest opinion on the mechanisms behind myopia development/progression, present further results from existing myopia interventions, and possibly introduce novel intervention methods.

\section{Advice for adopting myopia control in your practice}

When: now, even though we do not fully understand the mechanisms of some of the treatment strategies, and the success rate is not $100 \%$, the evidence shows simple strategies will have an effect in reducing the risk of myopic complications [6].

Who: all progressing myopes regardless of their baseline level of myopia. Also, children who have low levels of hypermetropia (>+0.75D) at age 6 , are more likely to become myopic [7]. It might be prudent to intervene before this occurs.

How: within the scope of refractive eye care practice, we would recommend fully correcting the refractive error with a multifocal soft contact lens design or recommending orthokeratology. The Proclear Multifocal (CooperVision) " $\mathrm{D}$ " design, with a $+2.00 \mathrm{D}$ add, is the only standard commercial lens published on to date [8]. Orthokeratology is a convenient option providing the patient with spectacle/contact lens-free vision during the day, however, it is more effective in patients with mildmoderate levels of myopia. In combination with myopia control intervention therapy, all patients should be advised to maximise time spent outdoors.

\section{References}

[1] K.L. Johnson, Are we myopic about myopia control? Contact Lens Anterior Eye 37 (4) (2014) 237-239.

[2] J.S. Wolffsohn, et al., Global trends in myopia management attitudes and strategies in clinical practice, Contact Lens Anterior Eye 39 (2) (2016) 106-116.

[3] D. Kurtz, et al., Role of parental myopia in the progression of myopia and its interaction with treatment in COMET children, Invest Ophthalmol Vis Sci 48 (2) (2007) 562-570.

[4] W.H. Chua, et al., Atropine for the treatment of childhood myopia, Ophthalmology 113 (12) (2006) 2285-2291.

[5] N.A. Brennan, Predicted reduction in high myopia for various degrees of myopia control, Contact Lens Anterior Eye 35 (2012) pe14-pe15. 
[6] D.I. Flitcroft, The complex interactions of retinal, optical and environmental factors in myopia aetiology, Prog. Retinal Eye Res. 31 (6) (2012) 622-660.

[7] K. Zadnik, et al., Prediction of juvenile-onset myopia, JAMA Ophthalmol. 133 (6) (2015) 683689.

[8] J.J. Walline, et al., Multifocal contact lens myopia control, Optom. Vis. Sci. 90 (11) (2013) 1207-1214.

[9] P. Kang, Acute and short-term changes in visual function with multifocal soft contact lens wear in young adults, Contact Lens Anterior Eye 39 (2) (2016) 133-140.

[10] S.J. Lee, Effect of orthokeratology in patients with myopic regression after refractive surgery, Contact Lens Anterior Eye 39 (2) (2016) 167-171.

[11] A. Mahomed, Structural design of contact lens-based drug delivery systems; in vitro and in vivo studies of ocular triggering mechanisms, Contact Lens Anterior Eye 39 (2) (2016) 97-105.

[12] M.K. Walker, Complications and fitting challenges associated with scleral contact lenses: a review, Contact Lens Anterior Eye 39 (2) (2016) 88-96.

[13] S.L.P. Weber, The use of ocular anatomical measurements using a rotating Scheimpflug camera to assist in the Esclera1 scleral contact lens fitting process, Contact Lens Anterior Eye 39 (2) (2016) 148-153.

[14] H. Li, The effects of hormone replacement therapy on dry eye syndromes evaluated by Schirmer test depend on patient age, Contact Lens Anterior Eye 39 (2) (2016) 124-127.

[15] J.M. Artigas, Light transmission and ultraviolet protection of contact lenses under artificial illumination, Contact Lens Anterior Eye 39 (2) (2016) 141-147.

[16] M. Boost, Determination of cytotoxicity of Traditional Chinese Medicine herbs, Rhizoma coptidis Radix scutellariae, and Cortex phellodendri, by three methods, Contact Lens Anterior Eye 39 (2) (2016) 128-132. 